\title{
Radiation Characteristics of Artificial Magneto- Dielectric Antenna Loaded with Split-Ring Resonator Array
}

\author{
Yohanes Galih Adhiyoga, Catur Apriono, Eko Tjipto Rahardjo \\ Department of Electrial Engineering, Universitas Indonesia, Depok City, West Java, Indonesia \\ Corresponding author: Eko Tjipto Rahardjo (e-mail: eko@eng.ui.ac.id).
}

\begin{abstract}
This study proposes a simple artificial magneto-dielectric (MD) antenna with a split-ring resonator (SRR) loaded in an array configuration. This SRR element is used to realize the MD characteristic that is beneficial for antenna miniaturization. The simulation of material permittivity and permeability is conducted to ensure that the proposed SRR-array configuration has the expected MD characteristic. Configurations of SRR in arrays of $3 \times 3,5 \times 5,7 \times 7$, and $9 \times 9$ are realized to obtain its radiation characteristics when embedded in the antenna ground plane. A rectangular patch antenna is used in this study. Based on the simulation, the proposed antenna with an SRR array of $5 \times 5$ achieves excellent MD characteristics with high permittivity and permeability of 32.44 and 51.39, respectively. Furthermore, a microstrip antenna loaded with the SRR $5 \times 5$ arrays are fabricated on a RT/duroid 5880 substrate. It has a dimension of $0.31 \lambda_{0} \times 0.26 \lambda_{0}$. The simulation and measurement results of the proposed antenna show a fractional bandwidth (FBW) of $4.79 \%$ and $5.2 \%$ at $1.5 \mathrm{GHz}$; and a maximum gain of $5.20 \mathrm{~dB}$ and $4.95 \mathrm{~dB}$, respectively. Good agreement between the simulated and the measured results was achieved.
\end{abstract}

INDEX TERMS magneto-dielectric antenna, split-ring resonator, radiation characteristics, miniaturization.

\section{INTRODUCTION}

The need for compact antennas in wireless communication systems has become a critical problem in recent times, with many researchers examining new and alternative materials for antenna miniaturization [1]. As magneto-dielectric (MD) material can strike an optimal balance between antenna size and performance, it is promising for miniaturization purposes [2]-[5]. Equation (1) shows that the antenna wavelength $\left(\lambda_{g}\right)$ that will determine the dimensions of the antenna is influenced by the relative dielectric permittivity $\left(\varepsilon_{r}\right)$ and relative magnetic permeability $\left(\mu_{r}\right)$ of the material [6].

$$
\lambda_{g}=\frac{c}{f_{o} \sqrt{\varepsilon_{r} \mu_{r}}}
$$

From Equation (1), it can be seen that the root of this multiplication of permittivity and permeability is often called refractive index (n) that is expressed in Equation (2).

$$
n=\sqrt{\mu_{r} \varepsilon_{r}}
$$

Equation (1) and (2) state that the higher the relative permittivity $\left(\varepsilon_{r}\right)$ and relative permeability $\left(\mu_{r}\right)$ of the material, the higher the refractive index $(n)$ or one can associate with miniaturization factor, and also the smaller the wavelength would be, which affects the smaller size of the antenna in the operating frequency $\left(f_{o}\right)$. These two properties can significantly affect patch dimension when the value of its parameter is nearly equal [7]-[9]. When the parameter is not balanced, it can affect the radiation characteristics resulting in insignificantly size reduction of the antenna. Unfortunately, the use of MD materials for antenna substrates is currently limited due to material scarcity. Any dielectric materials readily available in nature do not have magnetic properties $\left(\mu_{r}\right.$ $=1$ ), and conversely, readily available magnetic materials do not have dielectric properties $\left(\varepsilon_{r}=1\right)$. Accordingly, raw materials must first pass through a synthesis process in order to acquire expected MD characteristics; however, this process is complex and expensive. Therefore, various methods have been introduced in order to realize MD characteristics with simple processes by generating magnetic properties in dielectric materials, one of which is by adding inductive elements.

Marco et al. [10] develops a compact printed-dipole antenna using metamaterial loading as the inductive element to reduce the size to a minimum of $0.19 \lambda_{0} \times 0.04 \lambda_{0} \times 0.003 \lambda_{0}$ at 1.15 GHz. The negative refractive index of the metamaterial provides great flexibility with respect to controlling the permittivity and permeability when achieving miniaturization. The use of inductive loading to achieve dual-band and miniaturization has also been reported by [11] and [12] Homayoon et al. [11] uses a stub printed-tapered-monopole 
antenna, decreasing size to $0.07 \lambda_{0} \times 0.15 \lambda_{0}$ at a frequency of 1 GHz. Alternatively, Gupta et al. [12] combines the incorporated meandered lines, lumped capacitors, and volumetric reactive loadings in order to achieve size reduction, a high radiation efficiency, and dual-band response.

Using a transmission line model, as reported by [13], a size reduction of $74 \%$ compared to conventional antenna without loaded structure at a frequency of $2.62 \mathrm{GHz}$ can be obtained. The structure is achieved by introducing the capacitive and inductive loadings generated from the grounded strips and shorting pins. Moreover, [14] presents a planar artificial magnetic conductor (AMC) by combining capacitive and inductive loading to improve radiation performance, realizing a size reduction of $62.82 \%$ compared to the design without AMC surface. This AMC structure plays a role as a combination of the capacitive and inductive couplings to obtain effective material parameters.

In this study, the simple design of an artificial MD antenna is proposed, and its radiation characteristics will be examined. The aforementioned artificial magnetic structure is obtained by introducing a split-ring resonator (SRR) element as an inductive loading. The $3 \times 3,5 \times 5,7 \times 7$, and $9 \times 9$ SRR array configurations are designed to determine the generated effective material properties, which are represented by relative permittivity $\left(\varepsilon_{r}\right)$ and permeability $\left(\mu_{r}\right)$ parameters. Accordingly, full wave simulations of the permittivity and permeability responses are carried out using CST Microwave Studio. The antenna parameters analyzed in this work are Sparameters, gain, surface current, and antenna radiation efficiency. The antenna proposed herein is designed with a simple way of adopting the widely known SRR structure with an easy fabrication process.

\section{ARTIFICIAL MAGNETO-DIELECTRIC ANTENNA DESIGN}

\section{A. MATERIAL PROPERTIES}

To identify the permeability of the SRR element, simulations were conducted using the scattering-parameter extraction method. The SRR element was printed on the dielectric material as a radiating patch, which was fed with two waveguide ports on both sides; on the back side, a transmission line was printed on the substrate, as shown in Fig. 1. The dimensions of the SRR were obtained using equivalentcircuit models, following the work of [15]. This element was then simulated on RT/duroid 5880 substrate with a relative dielectric constant of 2.2 , a loss tangent of 0.0009 , and a thickness of $1.575 \mathrm{~mm}$ with the waveguide ports dimension of $49.38 \mathrm{~mm} \times 33.71 \mathrm{~mm}$. Material permittivity and permeability can be calculated from the refractive index ( $n$ ) and impedance $(z)$ according to Equation (3) and (4); likewise, $z$ is extracted from the transmission $\left(\mathrm{S}_{21}\right)$ and reflection $\left(\mathrm{S}_{11}\right)$ data, following the work of [16].

$$
\begin{gathered}
\varepsilon=\frac{n}{z} \\
\mu=n \times z
\end{gathered}
$$

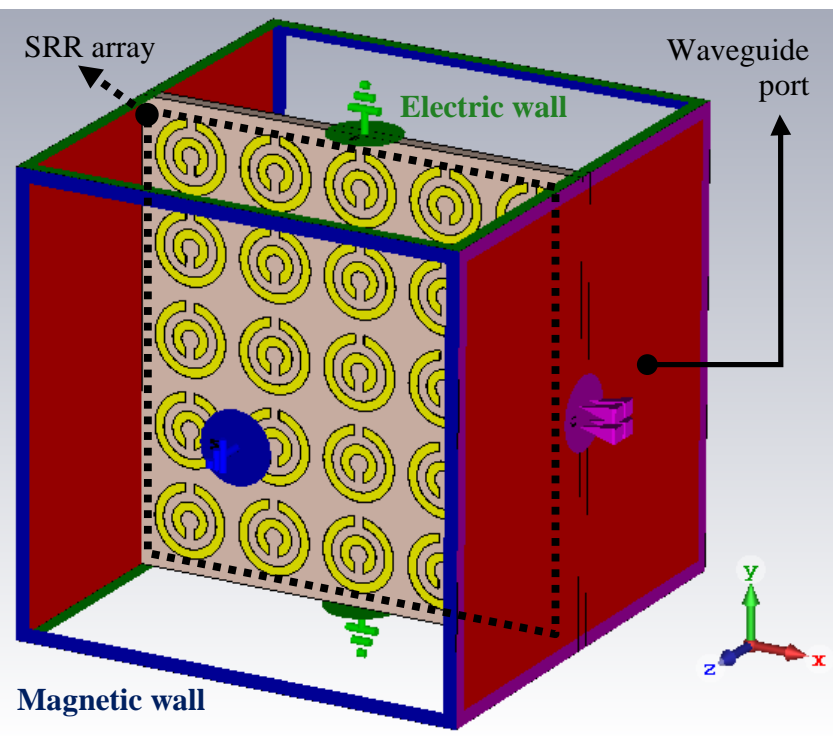

FIGURE 1. Set up of the effective material properties extraction over SRR $5 \times 5$ array.

To determine the effect of the SRR element on changes in refractive index, the arrays were arranged in $3 \times 3,5 \times 5,7 \times$ 7 and $9 \times 9$ configurations. Based on Fig. 2 it can be seen that as the SRR arrays increase, the refractive index $(n)$ will also increase. This is because with the increasing number of SRR elements, the capacitive and inductive properties generated will also increase. These capacitive and inductive properties represent the value of permittivity $\left(\varepsilon_{r}\right)$ and permeability $\left(\mu_{r}\right)$, respectively. From Fig. 2 one can see that these result agree with [17] who said that the area occupied by SRR can affect the permittivity and permeability, like their rectangular SRR design that have $\varepsilon_{r}$ and $\mu_{r}$ of 7.5 to 8, respectively. As in [18] the relative permittivity and permeability of 4.1 and 2.8 , can be obtained by modifying SRR dimension through the unit cell configuration. The shape of ELC and NB-SRR structure designed by [19] can also adjust the parameters to achieve high permittivity and permeability up to 25 and 15 , respectively. According to Equation (2), the increasing value of $\varepsilon_{r}$ and $\mu_{r}$ will also affect the increase in the value of refractive index.

\section{B. SPLIT-RING RESONATOR ARRAY PERFORMANCE}

To verify the effect of $n$ on antenna performance, a parametric study was also conducted on the effect of the number of SRR arrays on antenna performance. The aspects monitored were bandwidth, antenna gain, and efficiency. The proposed SRR arrays were in $3 \times 3,5 \times 5,7 \times 7$, and $9 \times 9$ configurations. The size of the radiating patch was designed with same dimensions for each SRR arrays. SRR array size can be adjusted according to the size of the radiating patch so that the occupied area of the SRR is just below the radiating patch. Table 1 shows the simulation results with respect to antenna performance for various SRR arrays.

From Table 1, it is evident that increasing the SRR array size results in greater FBW and higher efficiency. In addition, the gain parameter significantly changes with an increase in 


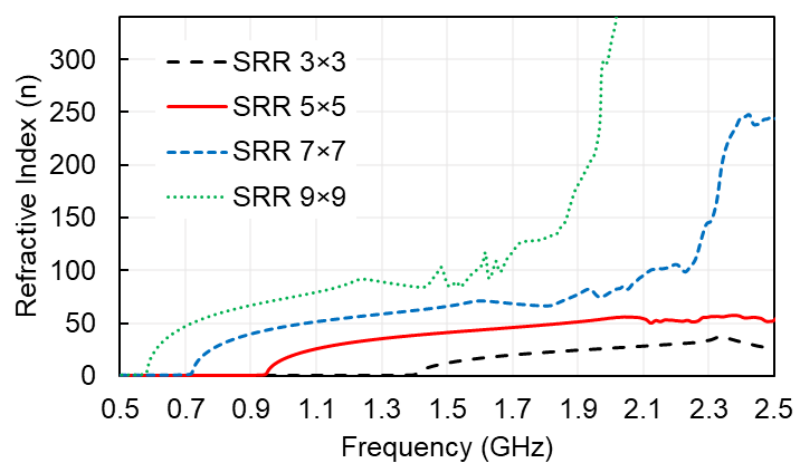

FIGURE 2. Simulation result of extracted refractive index on various SRR configurations.

TABLE I. Simulation results of antenna performance in various SRR array.

\begin{tabular}{cccc}
\hline \hline Configuration & FBW (\%) & Gain (dB) & Tot. Eff. (\%) \\
\hline SRR 3x3 & 3.23 & 5.28 & 92.54 \\
SRR 5x5 & 4.79 & 5.20 & 94.83 \\
SRR 7x7 & 6.19 & 5.37 & 95.60 \\
SRR 9x9 & 6.40 & 5.28 & 96.01 \\
\hline \hline
\end{tabular}

the SRR array size: the gain is relatively stable for various SRR array configurations. These results are promising with respect to improving bandwidth and antenna efficiency. Using an SRR $9 \times 9$ array design, FBW is expected to increase by a factor of up to 3.6; moreover, efficiency will improve by $14 \%$. However, antenna gain will slightly decrease because the power radiates in two opposite directions, reducing the magnitude of the main lobe.

From Fig. 2 and Table 1, it can be seen that the greater the number of SRR arrays, the greater the value of $n$ would be, and also, the higher value of $n$ will further increase the antenna performance. However, to obtain optimal antenna performance, one should consider embedding the SRR arrays as much as possible on the antenna ground plane. Table 1 indicates that identical trend achieved over the variation of the SRR arrays; therefore, with just single configuration sample, one can represent the effect of the number of SRR arrays on antenna performance. On the other hand, considering the fabrication capability of etching process in SRR elements and considering that SRR arrays of $7 \times 7$ and $9 \times 9$ have smaller dimensions, this study chooses the $5 \times 5$ SRR structure as a reference for design, parametric studies, fabrication, and measurements.

To ensure that an effective SRR array configuration has been obtained as element to embedded in antenna, the relative permittivity and permeability should be evaluated. The simulation results of the extracted permittivity and permeability are shown in Fig. 3. It can be seen that at the frequency of $1.5 \mathrm{GHz}$, the permittivity of 32.44 and permeability of 51.39 were obtained. These results also prove that at frequencies above $1 \mathrm{GHz}$ the SRR $5 \times 5$ configuration can be an effective medium with MD characteristics because it has $\varepsilon_{r}$ and $\mu_{r}$ of greater than unity.

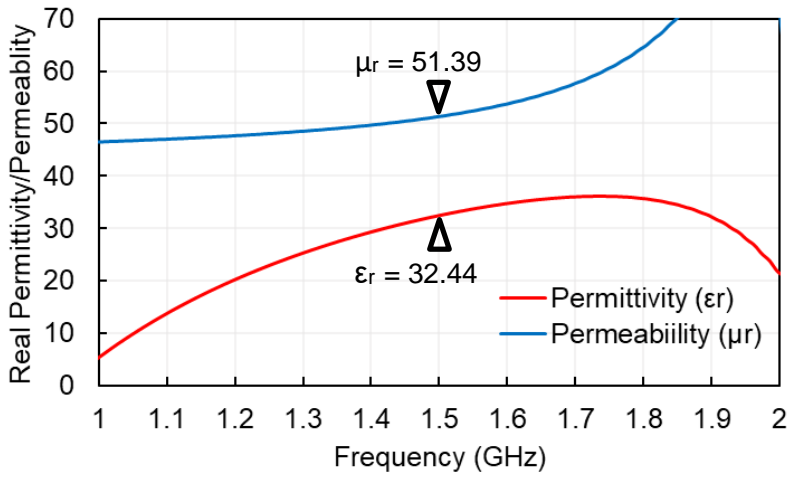

FIGURE 3. Extracted effective material properties of SRR $5 \times 5$ configuration.

\section{ANTENNA GEOMETRY}

After obtaining an SRR array design that meets the MD characteristics, the element was then combined with the microstrip patch antenna, which was embedded in the antenna ground plane. Fig. 4 (a) shows the top-view geometry of microstrip patch antenna fed by direct feeding line with inset and the proposed $5 \times 5$ SRR array configuration (Fig. 4 (b)). The SRR parameters considered in the design optimization consisted of the following: ring radius $(R=4 \mathrm{~mm})$, distance between the rings $(G a p=1 \mathrm{~mm})$, ring width $\left(W_{r}=1 \mathrm{~mm}\right)$, distance between SRR $(l=1.562 \mathrm{~mm})$, and slot width $\left(L_{b}=1\right.$ $\mathrm{mm})$. The dimensions of the microstrip patch antenna consisted as following: $W_{s}=112.41 \mathrm{~mm}, L_{s}=102.49 \mathrm{~mm}, W p$ $=62.41 \mathrm{~mm}, L p=52.49 \mathrm{~mm}, W_{f}=4.89 \mathrm{~mm}, W_{i}=1.2225 \mathrm{~mm}$, and $L_{i}=20 \mathrm{~mm}$.

The SRR element has two main parts: the inner ring and the outer ring. These are separated by a certain distance (Gap), as shown in Fig. 4. The SRR element was arranged in an array configuration to fulfill the radiating patch area. Since the size of SRR array was much smaller than the wavelength at the resonant frequency, and since it was placed directly underneath the radiating patch, the SRR element was able to become an effective MD medium [20]. Therefore, even though the SRR number increases, the SRR array size must meet the radiating patch size.

\section{PARAMETRIC STUDIES}

Many parameters were involved in the design of the single SRR element for this proposed antenna. The equivalent model of the SRR element consists of inductive $(L)$ and capacitive $(C)$ components. The $L$ component can be identified from the copper length denoted by the ring radius $(R)$ and the distance between rings (Gap), whereas the slot width $\left(L_{b}\right)$ and ring width $\left(W_{r}\right)$ is a capacitive component. Each parameter affects the antenna performance because of the changes in permittivity and permeability. Accordingly, in this section, the effect of several parameters on material permeability is reviewed by fixing one parameter and varying the others. The single element of SRR structure is chosen to investigate that effect on each parameter. Fixed parameters are denoted by solid red lines, as shown in Fig. 5. Therefore, the contribution of each parameter to the magnetic properties of the SRR element can be identified. 


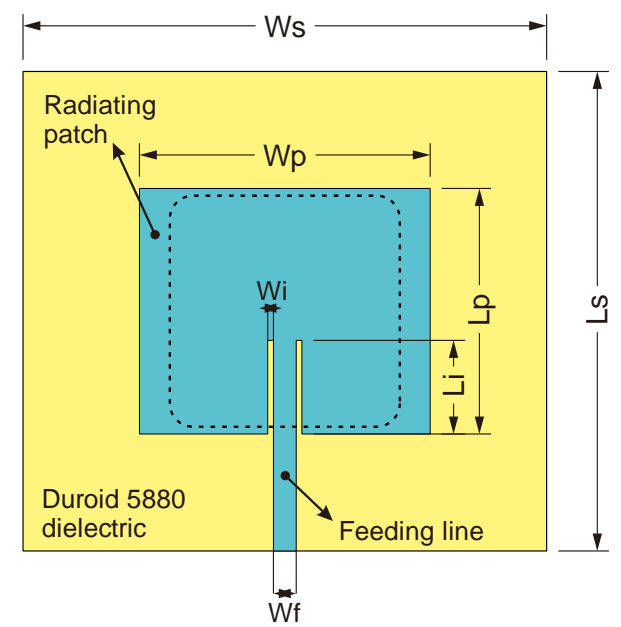

(a)

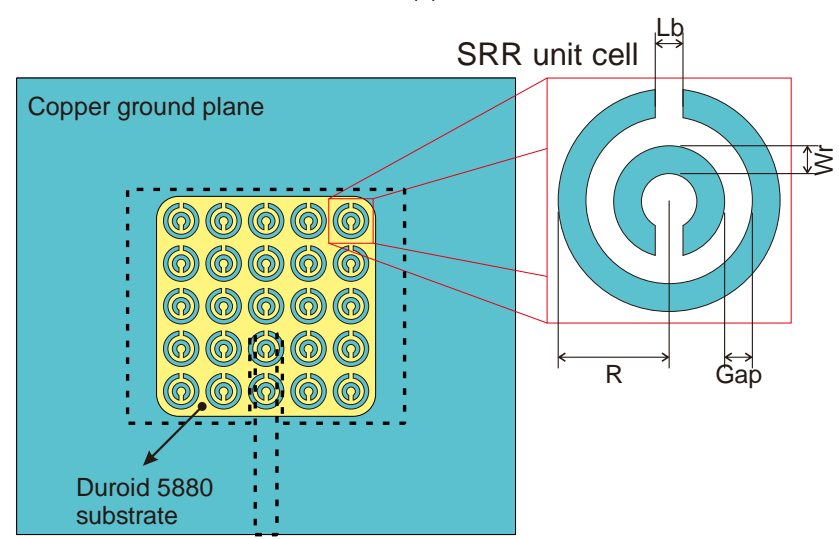

(b)

FIGURE 4. The microstrip antenna illustration with the proposed SRR array loading: (a) Top view; (b) bottom view.

Based on the parametric results, $R$ and Gap is the most influential parameter with respect to varying the value of material permeability in the working frequency: permeability decreases with an increase in $R$ (Fig. 5 (a)) and Gap (Fig. 5 (c)). Moreover, $L_{b}$ does not significantly affect permeability: changes in $L_{b}$ do not affect the size of the SRR itself, so it is shown that just a little increase in permeability when increasing the $L_{b}$ (Fig. 5 (b)). As expected, the similar response can be seen when $W_{r}$ is changed. That is almost no changes in permeability at frequency range of $1.3-1.5 \mathrm{GHz}$ (Fig. 5 (d)). $R$ and Gap parameters provide the inductive properties of the element, which increases permeability. While the $L_{b}$ and $W_{r}$ provide the capacitive properties that contributes in increasing the permittivity. This increase will affect the value of $n$, where this value will be maximum when the permeability drops sharply at approximately $1.85 \mathrm{GHz}$ as shown in Fig. 5.

\section{EXPERIMENTAL RESULTS}

The proposed antenna prototype is fabricated and measured to validate the simulated results, as shown in Fig. 6 and Fig. 7. The measurements include the reflection coefficient, antenna gain, and radiation pattern. The reflection coefficient was measured using Rohde Schwarz VNA, whereas the antenna

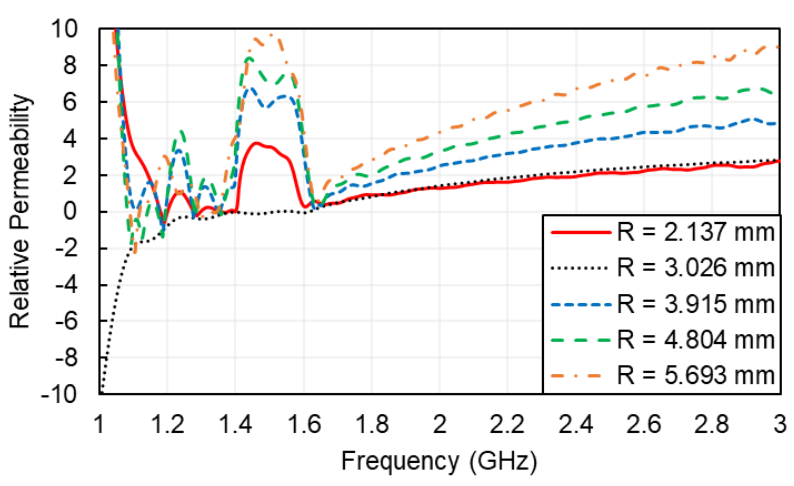

(a)

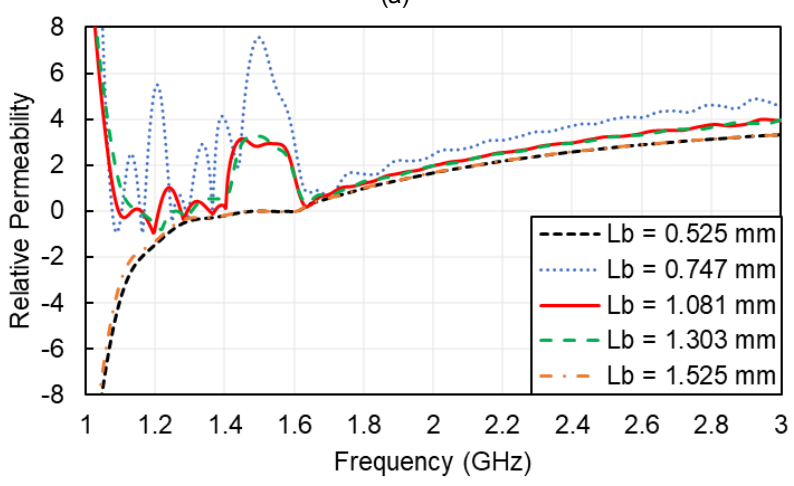

(b)

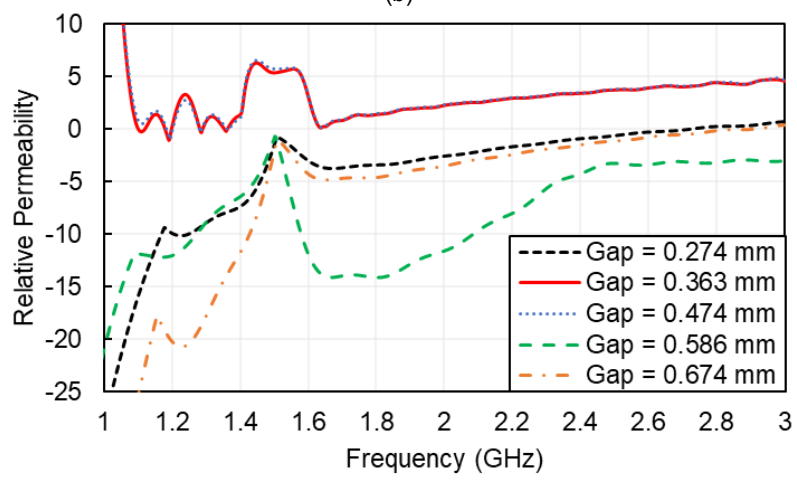

(c)

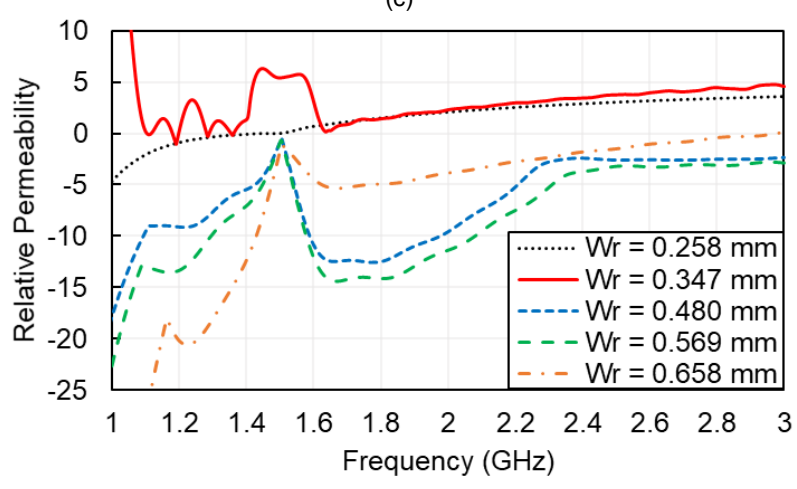

(d)

FIGURE 5. Parametric study on SRR parameters that affect permeability: (a) $R$, (b) $L_{b}$, (c) Gap, and (d) $W_{r}$. 


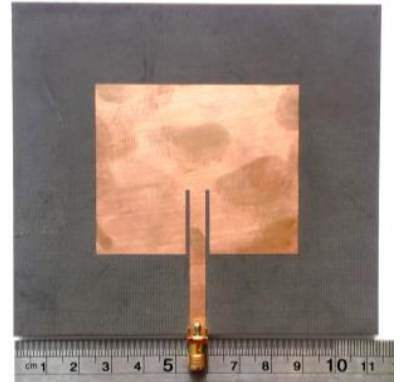

(a)

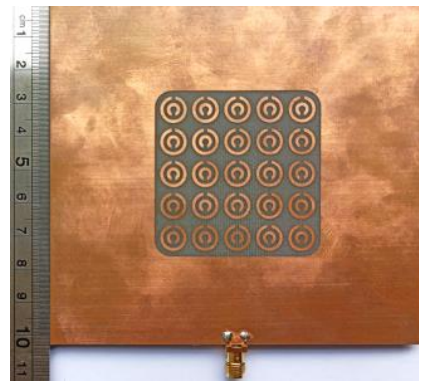

(b)
FIGURE 6. Photographs of the fabricated antenna with SRR $5 \times 5$ array configuration: (a) front view, (b) back view.

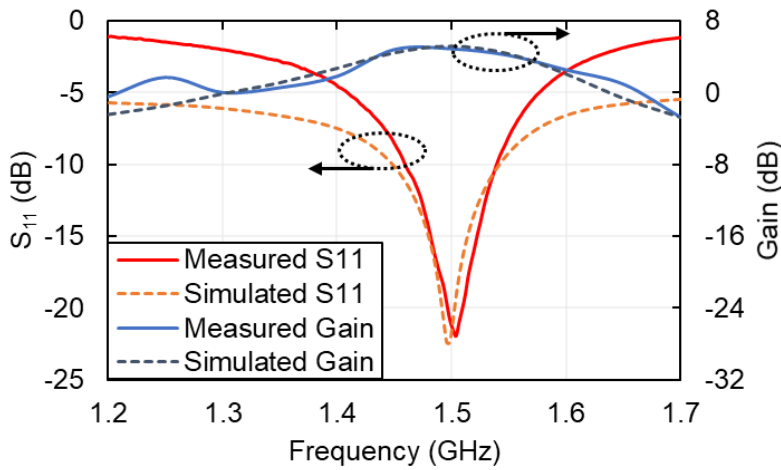

FIGURE 7. Simulated and measured S-parameters and gain of SRR $5 \times 5$ antenna.

gains and radiation pattern measurements were obtained using a gain comparison method, which uses the BBHA 9120A horn antenna as a reference.

According to Fig. 7, the results of the simulated and measured reflection coefficient and antenna gain are consistent with each other. The measured fractional bandwidth (FBW) and antenna gain are 5.2\% and $4.95 \mathrm{~dB}$, respectively. To investigate the effect of SRR array on the working mechanism of a MD antenna, simulations on the current distribution were carried out, as shown in Fig. 8. It can be seen from Fig.8 (a), it is evident that the maximum current mostly radiates through the rectangular side separating the ground plane and SRR element, and it partly radiates through the surface of the SRR element. As a result, the radiating patch did not radiate current as much as the antenna without an SRR array, since the power radiated through the radiating patch (Fig. 8 (b)). This is why the radiation pattern of the antenna with an SRR array exhibits dual-beam directions, both from the radiating patch and the SRR array.

The simulations and measurements of normalized radiation patterns are illustrated in Fig. 9. The E-plane $\left(\mathrm{phi}=0^{\circ}\right.$ ) pattern shows the magnitude differences in the directions of $90^{\circ}$ and $270^{\circ}$. It happens because there is a ratio difference between the maximum and minimum of measured radiated power that is more significant than the difference between the maximum and minimum of simulated results (Fig. 9 a). Good agreement is observed between the simulation and measurement of $\mathrm{H}$ plane $\left(\mathrm{phi}=90^{\circ}\right.$ ) pattern, particularly in the main lobe; conversely in the back lobe, there is little difference owing to

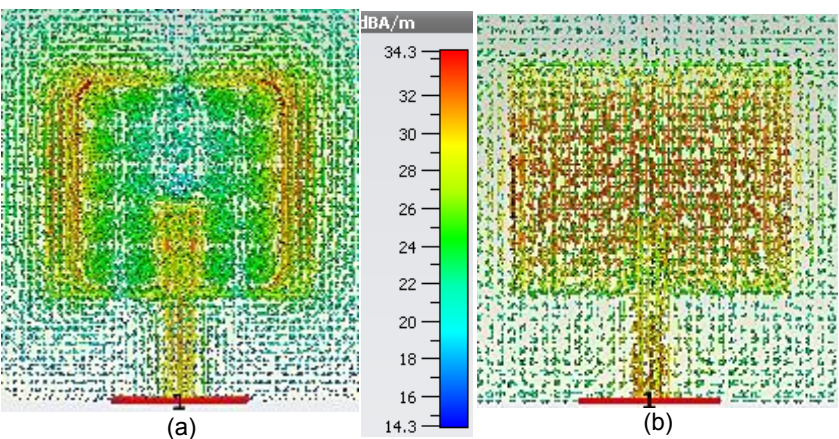

FIGURE 8. Current distributions of the two antennas (a) with SRR $5 \times 5$ and (b) without SRR at $1.5 \mathrm{GHz}$.

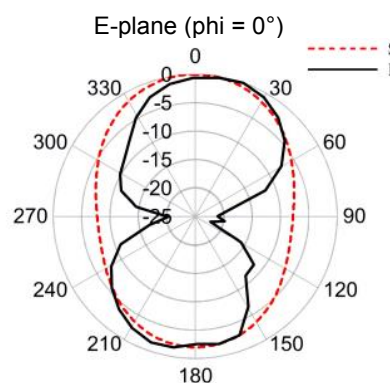

(a)

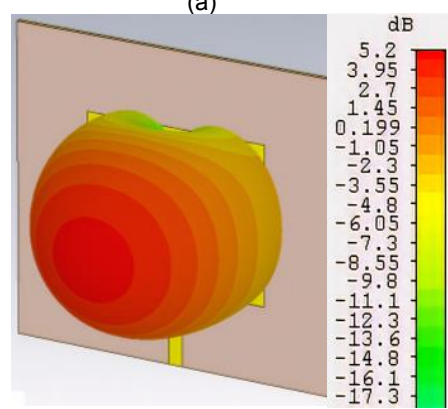

(c)

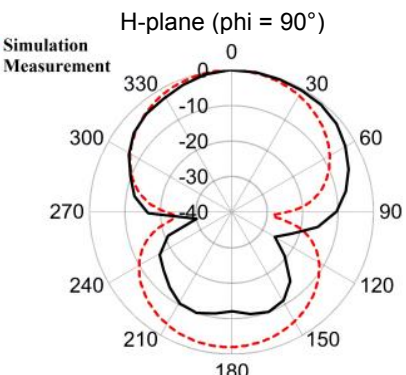

(b)
FIGURE 9. Current distributions of the two antennas (a) with SRR $5 \times 5$ and (b) without SRR at $1.5 \mathrm{GHz}$.

the diffraction factors that were not considered during the simulation (Fig. 9 b). As seen in Fig. 9 (c) and (d), the SRR array has uncommon radiation patterns compared with the conventional microstrip antennas which usually has a unidirectional radiation pattern. In the proposed antenna, an additional beam is present in the opposite direction of the main lobe. Nevertheless, as seen in Fig. 9 (c) radiation with a stronger magnitude (maximum gain: $5.2 \mathrm{~dB}$ ) is still occurs at the broadside of the operation band, as expected.

The proposed antenna design with SRR array configuration to realize the MD characteristics has advantages in bandwidth and radiation efficiency compared to some references performed by other researchers. An overview of antenna performance from several literature and its comparison with the proposed SRR array configuration attached on microstrip antenna can be seen in Table 2. Based on Table 2, the proposed antenna has higher bandwidth than [3], [10], [13], and [21]. It also has a bandwidth and efficiency of $0.6 \%$ and $22.83 \%$ higher than [22] that uses the similar SRR configuration. 
TABLE II. Overview of antenna radiation performance using various method compared to proposed antenna.

\begin{tabular}{|c|c|c|c|c|c|c|}
\hline Reference & $\begin{array}{c}\text { Frequency } \\
(\mathrm{GHz})\end{array}$ & $\begin{array}{l}\text { Dimension } \\
(\mathrm{mm})\end{array}$ & $\begin{array}{c}\text { Bandwidth } \\
(\%)\end{array}$ & $\begin{array}{l}\text { Gain } \\
(\mathrm{dB})\end{array}$ & $\begin{array}{c}\text { Efficiency } \\
(\%)\end{array}$ & Method \\
\hline$[3]$ & 2.48 & $0.20 \lambda_{0} \times 0.14 \lambda_{0}$ & 0.48 & 3.20 & 45.00 & metallic vias and strips \\
\hline$[10]$ & 1.15 & $0.19 \lambda_{0} \times 0.04 \lambda_{0}$ & 3.21 & 0.11 & 49.50 & $\begin{array}{l}\text { capacitive gaps and shunt inductive } \\
\text { strips }\end{array}$ \\
\hline$[13]$ & 2.62 & $0.087 \lambda_{0} \times 0.192 \lambda_{0}$ & 1.60 & 3.90 & 88.10 & $\begin{array}{l}\text { capacitive and inductive loadings } \\
\text { (grounded strips) }\end{array}$ \\
\hline$[21]$ & 2.35 & $0.12 \lambda_{0} \times 0.17 \lambda_{0}$ & 0.89 & 1.34 & 48.05 & metallic inclusion \\
\hline$[22]$ & 1.80 & $0.24 \lambda_{0} \times 0.24 \lambda_{0}$ & 4.60 & 6.24 & 72.00 & spiral loop resonator SRR \\
\hline$[23]$ & 2.45 & $0.32 \lambda_{0} \times 0.32 \lambda_{0}$ & 6.65 & 6.89 & 88.87 & multi square loop meta-substrate \\
\hline Proposed & 1.50 & $0.31 \lambda_{0} \times 0.26 \lambda_{0}$ & 5.22 & 4.95 & 94.83 & SRR Array \\
\hline Conventional & 1.50 & $0.34 \lambda_{0} \times 0.33 \lambda_{0}$ & 0.69 & 6.27 & 76.09 & without SRR \\
\hline
\end{tabular}

\section{CONCLUSION}

In this study, an artificial MD antenna realized by embedding SRR array on patch antenna was designed, fabricated, measured, and its radiation characteristics performance was evaluated. Based on the measurements, the proposed antenna loaded with an SRR $5 \times 5$ arrays could achieve an FBW of $5.2 \%$ and a gain of $4.95 \mathrm{~dB}$ at a frequency of $1.5 \mathrm{GHz}$. The fabricated antenna has an acceptable size of $0.31 \lambda_{0} \times 0.26 \lambda_{0}$, making it $28.42 \%$ smaller than the conventional antenna. Furthermore, the simplicity of the SRR array design in producing MD characteristics makes this method appropriate for applications that require the reduced size of an antenna that works at lower frequency without deteriorating their performances. It is also possible to combine this SRR structure with the pure magneto-dielectric material to obtain better antenna radiation characteristics.

\section{ACKNOWLEDGMENT}

This work was supported in part by the Ministry of Research, Technology and Higher Education, Indonesian Government in terms of Penelitian Pendidikan Magister menuju Doktor untuk Sarjana Unggul (PMDSU) under Grant No: NKB1858/UN2.R3.1/HKP.05.00/2019.

\section{REFERENCES}

[1] M. Fallahpour and R. Zoughi, “Antenna Miniaturization Techniques: A Review of Topology- and Material-Based Methods," IEEE Antennas Propag. Mag., 2018.

[2] P. M. T. Ikonen, K. N. Rozanov, A. V Osipov, P. Alitalo, and S. A. Tretyakov, "Magnetodielectric Substrates in Antenna Miniaturization: Potential and Limitations," IEEE Trans. Antennas Propag., vol. 54, no. 11, pp. 3391-3399, 2006.

[3] F. Farzami, K. Forooraghi, and M. Norooziarab, "Miniaturization of a Microstrip Antenna Using a Compact and Thin Magneto-Dielectric Substrate," IEEE Antennas Wirel. Propag. Lett., vol. 10, pp. 15401542, 2011.

[4] J. Lee, J. Lee, K. Min, and Y. Cheon, "Miniaturized Antennas With Reduced Hand Effects in Mobile Phones Using Magneto-Dielectric Material," IEEE Antennas Wirel. Propag. Lett., vol. 13, pp. 935-938, 2014.
[5] K. Han et al., "Magneto-Dielectric Nanocomposite for Antenna Miniaturization and SAR Reduction," IEEE Antennas Wirel. Propag. Lett., vol. 15, pp. 72-75, 2016.

[6] A. Foroozesh and L. Shafai, "Size reduction of a microstrip antenna with dielectric superstrate using meta-materials: Artificial magnetic conductors versus magneto-dielectrics," in Proc. IEEE Int. Symp, 2006, pp. 11-14.

[7] L. B. Kong, Z. W. Li, G. Q. Lin, and Y. B. Gan, "Ni-Zn Ferrites Composites With Almost Equal Values of Permeability and Permittivity for Low-Frequency Antenna Design," in IEEE Transactions on Magnetics, 2007.

[8] Z. Zheng, H. Zhang, J. Q. Xiao, and F. Bai, "Low Loss $\mathrm{NiZn/Co} 2 \mathrm{Z}$ Composite Ferrite With Almost Equal Values of Permeability and Permittivity for Antenna Applications," IEEE Trans. Magn., vol. 49, no. 7, pp. 4214-4217, 2013.

[9] T. Zhang, H. Su, X. Tang, H. Zhang, Y. Jing, and B. Liu, "Low-Loss Composite NiCuZnCo-BaTiO3 With Matched Permeability and Permittivity in High Frequency Range," IEEE Trans. Magn., vol. 50, no. 11, pp. 1-4, 2014.

[10] M. A. Antoniades and G. V. Eleftheriades, "Multiband compact printed dipole antennas using NRI-TL metamaterial loading," IEEE Trans. Antennas Propag., 2012.

[11] H. Oraizi and B. Rezaei, "Combline loadings of printed triangular monopole antennas for the realization of multi-band and wideband characteristics," Prog. Electromagn. Res. B, 2012.

[12] S. Gupta and G. Mumcu, "Dual-band miniature coupled double loop GPS antenna loaded with lumped capacitors and inductive pins," IEEE Trans. Antennas Propag., 2013.

[13] M. Yang, Z. N. Chen, P. Y. Lau, X. Qing, and X. Yin, "Miniaturized patch antenna with grounded strips," IEEE Trans. Antennas Propag., 2015.

[14] H. Malekpoor and S. Jam, "Improved Radiation Performance of Low Profile Printed Slot Antenna Using Wideband Planar AMC Surface," IEEE Trans. Antennas Propag., 2016.

[15] J. D. Baena et al., "Equivalent-circuit models for split-ring resonators and complementary split-ring resonators coupled to planar transmission lines," IEEE Trans. Microw. Theory Tech., 2005.

[16] X. Chen, T. M. Grzegorczyk, B. I. Wu, J. Pacheco, and J. A. Kong, "Robust method to retrieve the constitutive effective parameters of metamaterials," Phys. Rev. E - Stat. Physics, Plasmas, Fluids, Relat. 
Interdiscip. Top., 2004.

[17] Y. Liu, R. Raju, L. Shafai, and C. Shafai, "A miniaturized artificial magneto-dielectric resonator antenna with split ring resonators," in Antenna Technology and Applied Electromagnetics (ANTEM), 2016 17th International Symposium on, 2016, pp. 1-2.

[18] J. Zaid, M. Mantash, A. Kesavan, and T. A. Denidni, "Miniaturized Microstrip Patch Antenna Using Magneto-dielectric Substrate for RFID applications," in 2018 IEEE Antennas and Propagation Society International Symposium and USNC/URSI National Radio Science Meeting, APSURSI 2018 - Proceedings, 2018.

[19] Q. Nguyen and A. I. Zaghloul, "Impedance Matching Metamaterials Composed of ELC and NB-SRR," in 2018 IEEE Antennas and Propagation Society International Symposium and USNC/URSI National Radio Science Meeting, APSURSI 2018 - Proceedings, 2018.

[20] X. M. Yang et al., "Increasing the Bandwidth of Microstrip Patch Antenna by Loading Compact Artificial Magneto-Dielectrics," IEEE Trans. Antennas Propag., vol. 59, no. 2, pp. 373-378, 2011.

[21] S. Kumar and D. K. Vishwakarma, "Miniaturisation of microstrip patch antenna using an artificial planar magneto-dielectric metasubstrate," IET Microwaves, Antennas Propag., vol. 10, no. 11, pp. 1235-1241, 2016.

[22] K. Buell, H. Mosallaei, and K. Sarabandi, "A substrate for small patch antennas providing tunable miniaturization factors," IEEE Trans. Microw. Theory Tech., vol. 54, no. 1, pp. 135-146, 2006.

[23] A. Pinsakul, P. Supanakoon, and S. Promwong, "Performance Evaluation of Artificial Megneto-Dielectric Metamaterial with Microstrip Patch Antenna for Wireless System," in 6th Global Wireless Summit, GWS 2018, 2018. 